\title{
Apparent transverse relaxation rates in systems with coupled carbon-13 spins
}

\author{
Takuya F. Segawa, ${ }^{a}$ Nicolas Aeby $^{a}$ and Geoffrey Bodenhausen* ${ }^{a b}$ \\ Received 16th March 2010, Accepted 13th May 2010 \\ DOI: $10.1039 / \mathbf{c 0 0 4 2 9 3 e}$
}

In systems with homonuclear scalar couplings, the envelopes of spin echoes obtained with simple refocusing pulses or trains of such pulses are normally modulated so that it is difficult to extract transverse relaxation rates. It has been shown recently that echo modulations can be quenched by cumulative pulse errors that arise after applying a large number of refocusing pulses with moderate $r f$ amplitudes. The resulting unmodulated decays allow one to extract apparent transverse relaxation rates. Early work on systems comprising only two nitrogen-15 nuclei or two carbon-13 spins has recently been extended to systems with coupled protons. This work focuses on systems with three coupled carbon-13 spins, which in turn are coupled to several neighbouring protons. Unmodulated echo trains can be obtained by optimizing the pulse interval, the carrier frequency and the $r f$ amplitude of the refocusing pulses.

\section{Introduction}

The measurement of transverse relaxation rates $R_{2}=1 / T_{2}$ of various nuclei can provide valuable information about internal dynamics. ${ }^{1}$ Spin echo sequences with multiple refocusing pulses ${ }^{2,3}$ are commonly used to extract $R_{2}$ rates of nitrogen- 15 nuclei. In proteins, these nuclei are isolated from each other, in the sense that scalar couplings $J_{\mathrm{NN}}$ between amide nitrogen-15 nuclei can be neglected. In nucleic acids however, $J_{\mathrm{NN}}$ couplings in fully nitrogen- 15 enriched aromatic purine and pyrimidine systems and across hydrogen bonds lead to modulations of echo trains. Likewise, homonuclear scalar $J_{\mathrm{CC}}$ couplings tend to mask the envelopes of echo decays in fully carbon-13 enriched molecules. Needless to say, the determination of transverse relaxation of protons is challenging in virtually all molecules because echoes are invariably modulated by scalar $J_{\mathrm{HH}}$ couplings.

It has recently been shown that under certain conditions, it is possible to quench echo modulations due to homonuclear scalar couplings. ${ }^{4-9}$ In two-spin systems, so-called apparent transverse relaxation rates $R_{2}^{\text {app }}$ can be extracted from echo decays provided that they do not suffer from significant modulations. Similar observations have been made in proton spectra of cyclosporin, which features extensive networks of coupled spins. ${ }^{10}$

A multiple-refocusing Carr-Purcell-Meiboom-Gill (CPMG) sequence ${ }^{2,3}$ can be written:

$$
(\pi / 2)_{y}-\left(\tau-\pi_{x}-\tau\right)_{2 n}
$$

\footnotetext{
${ }^{a}$ Institut des Sciences et Ingénierie Chimiques,

Ecole Polytechnique Fédérale de Lausanne, $B C H$,

1015 Lausanne,Switzerland. E-mail: geoffrey.bodenhausen@epfl.ch;

Fax: + 41 (0)21693 94 35; Tel: + 41 (0)216939431

${ }^{b}$ Département de Chimie, associé au CNRS, Ecole Normale

Supérieure, 24 Rue Lhomond, 75231 Paris Cedex 05, France.

Tel: $+33(0) 144323344$
}

We shall only consider even echoes with $2 n=2,4,6 \ldots$ The duration of each CPMG cycle is

$$
\tau_{\mathrm{c}}=\left[4 \tau+2 \tau_{\pi}\right]
$$

where $\tau_{\pi}$ is the duration of a $\pi$ pulse.

In a frame rotating at the carrier frequency $\omega_{r f}$, the Hamiltonian for a system with three spins $I=\frac{1}{2}, S=\frac{1}{2}$, and $R=\frac{1}{2}$ (e.g., three carbon-13 nuclei) may be written:

$$
\begin{aligned}
H= & \Omega_{I} I_{z}+\Omega_{S} S_{z}+\Omega_{R} R_{z}+2 \pi J_{I S} \boldsymbol{I} \cdot \boldsymbol{S} \\
& +2 \pi J_{I R} \boldsymbol{I} \cdot \boldsymbol{R}+2 \pi J_{S R} \boldsymbol{S} \cdot \boldsymbol{R}
\end{aligned}
$$

with offsets $\Omega_{I}=\omega_{0 I}-\omega_{r f}, \Omega_{R}=\omega_{0 R}-\omega_{r f}$, and $\Omega_{S}=$ $\omega_{0 S}-\omega_{r f}$, where $\omega_{0 I}$ is the Larmor frequency of spin $I$ in the laboratory frame.

Note that heteronuclear couplings are not included in this Hamiltonian, i.e., we assume that the protons are decoupled throughout. In actual fact, decoupling during multiple refocusing sequences may be challenging, for there can be complicated interference effects between the CPMG cycle $\tau_{\mathrm{c}}$ and the cycle (or supercycle) of a (composite pulse) decoupling sequence. Even continuous-wave $(\mathrm{CW})$ proton decoupling with a constant $r f$ amplitude $\omega_{1}\left({ }^{1} \mathrm{H}\right)$, which has a cycle $\tau_{\mathrm{cw}}=2 \pi / \omega_{1}\left({ }^{1} \mathrm{H}\right)$, can interfere with multiple refocusing of nuclei such as ${ }^{13} \mathrm{C},{ }^{15} \mathrm{~N}$, etc. These interference effects can accelerate the decay of echo trains, and thus increase the apparent transverse relaxation rates $R_{2}{ }^{\text {app }}$. A detailed discussion of decoupling effects is beyond the scope of this work. A safe way to circumvent these complications is to avoid proton decoupling altogether during the multiple refocusing sequence, so that the decoupler is activated only during signal acquisition, i.e., during the second half of the $2 n^{\text {th }}$ echo. In the absence of decoupling, the signals of a spin $S$ are split into multiplets by heteronuclear couplings $J_{S K}$ with protons $K$. The behaviour of each separate multiplet component under the multiple refocusing sequence can be understood if we consider that it has a 
different effective offset. Thus, in a system with a single proton $K$, we must consider two distinct offsets $\Omega_{S}+\pi J_{S K}$ and $\Omega_{S}-\pi J_{S K}$, instead of a single offset at the chemical shift $\Omega_{S}$. In ${ }^{13} \mathrm{C}$ spectra of alanine $\left(\mathrm{NH}_{3}{ }^{+} \mathrm{CHCH}_{3} \mathrm{COO}^{-}\right)$, one must also consider degenerate heteronuclear couplings that lead to quartets.

The homonuclear scalar couplings in eqn (3) have the full isotropic form,

$$
\boldsymbol{I} \cdot \boldsymbol{S}=I_{x} S_{x}+I_{y} S_{y}+I_{z} S_{z}
$$

If the refocusing pulses are very far off-resonance, the coherences precess in each cycle $\tau_{\mathrm{c}}$ under the effect of the offsets so that they accumulate phase shifts $\Phi$,

$$
\Phi_{I}=\Omega_{I} \tau_{\mathrm{c}}, \Phi_{S}=\Omega_{S} \tau_{\mathrm{c}}, \Phi_{R}=\Omega_{R} \tau_{\mathrm{c}}
$$

For a system with three spins $I, S$, and $R$ the carrier frequency $\omega_{r f}$ can be set on resonance for spin $I\left(\Omega_{I}=0\right)$. The tilt angles $\theta_{S}$ and $\theta_{R}$ of the effective fields experienced by the two other spins during refocusing pulses with an $r f$ amplitude $\omega_{1}$ can be described by the tilt parameters:

$$
\gamma_{S}=\Omega_{S} / \omega_{1}, \gamma_{R}=\Omega_{R} / \omega_{1} .
$$

The analysis of the full CPMG cycle $^{8}$ shows that the modulations of the echoes are most pronounced when

$$
\begin{gathered}
\Phi_{S}^{\prime \prime}=\Omega_{S} \tau_{\mathrm{c}}-\pi \gamma_{S}=4 k_{S} \pi \\
\Phi_{R}^{\prime \prime}=\Omega_{R} \tau_{\mathrm{c}}-\pi \gamma_{R}=4 k_{R} \pi
\end{gathered}
$$

with integers $k_{S}$ and $k_{R}$. When either of these conditions is satisfied, the scalar couplings $J_{I S}$ or $J_{I R}$ are said to be 'recoupled', so that the echoes are fully modulated. These conditions have superficial similarities with sidebands due to delays alternating with nutation for tailored excitation (DANTE). ${ }^{11,12}$ We shall speak of sidebands of order $k$, or simply of the $k^{\text {th }}$ sideband. In a three-spin system with $\Omega_{I}=0$, there are two distinct conditions where either $J_{I S}$ or $J_{I R}$ are 'recoupled' and lead to echo modulations: ${ }^{9}$

$$
\begin{gathered}
\tau_{S}=\frac{\pi\left(4 k_{S} \omega_{1}-\Omega_{S}\right)}{4 \Omega_{S} \omega_{1}} \\
\tau_{R}=\frac{\pi\left(4 k_{R} \omega_{1}-\Omega_{R}\right)}{4 \Omega_{R} \omega_{1}}
\end{gathered}
$$

The intervals between these recoupling conditions are inversely proportional to the corresponding offsets:

$$
\begin{aligned}
& \Delta \tau_{S}=\pi / \Omega_{S} \\
& \Delta \tau_{R}=\pi / \Omega_{R}
\end{aligned}
$$

These echo modulations can be simulated numerically by calculating the expectation values $\left\langle I_{x}\right\rangle$ at the top of the $2 n^{\text {th }}$ echo (there are no phase-shifts, so that $\left\langle I_{y}\right\rangle=0$ if $\Omega_{I}=0$ ), considering the full Hamiltonian without relaxation effects. The amplitude of the echo modulations of spin $I$ is defined over the relevant range of refocusing pulses.

$$
A_{\text {mod }}=\frac{\max \left(\left\langle I_{x}\right\rangle\right)-\min \left(\left\langle I_{x}\right\rangle\right)}{2}
$$

We shall begin by simulating the behavior of a simple twospin system. Fig. 1 shows how the amplitude $A_{\text {mod }}$ of the

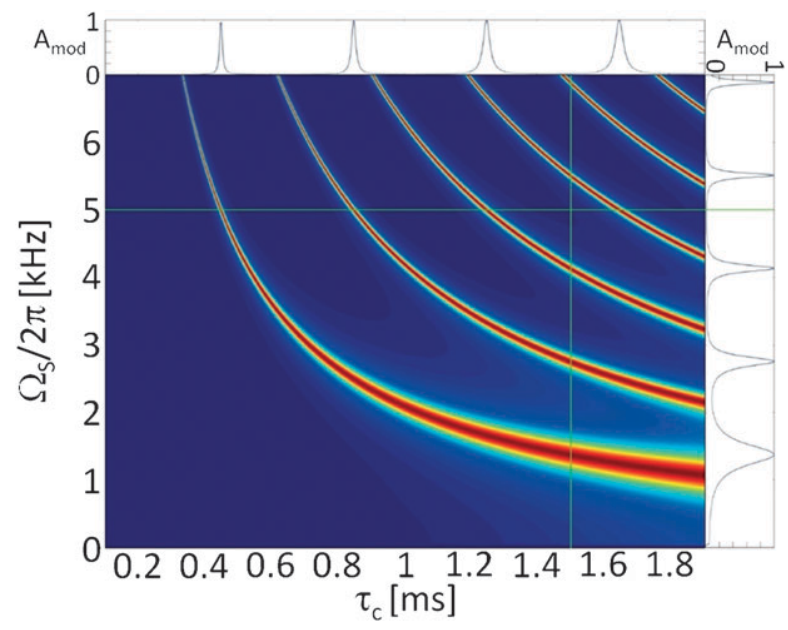

Fig. 1 Amplitudes $A_{\text {mod }}$ of echo modulations calculated for a twospin system as a function of the offset $\Omega_{S}$ of the coupling partner $S$ when $\Omega_{I}=0$, for a CPMG cycle of length $\tau_{\mathrm{c}}=\left[4 \tau+2 \tau_{\pi}\right]$ and an $r f$ amplitude $\omega_{1} /(2 \pi)=10 \mathrm{kHz}$. Dark blue areas correspond to regions without modulations, whereas red ridges indicate maximum recoupling conditions. The plot along the top shows $A_{\bmod }$ as a function of $\tau_{\mathrm{c}}$ for a fixed offset $\Omega_{S} /(2 \pi)=5 \mathrm{kHz}$, while the plot along the right side shows $A_{\text {mod }}$ as a function of the offset $\Omega_{S}$ of the second spin for a fixed $\tau_{\mathrm{c}}=1.5 \mathrm{~ms}$.

modulations of the expectation value $\left\langle I_{x}\right\rangle$ depends on the offset $\Omega_{S}$ of the coupling partner and on the cycle length $\tau_{\mathrm{c}}$, or, equivalently, on the duration of the defocusing and refocusing intervals $\tau$. Sharp recoupling conditions are observed for short cycle lengths $\tau_{\mathrm{c}}$ and for large offsets $\Omega_{S}$, when the effective fields are strongly tilted, i.e., for large values of $\gamma_{S}$. On the other hand, small $\gamma_{S}$ ratios lead to broader recoupling conditions.

In systems with three or more spins, one observes a superposition of different recoupling conditions characterized by different widths. Instead of showing extensive simulations of systems with $N$ spins in multidimensional parameter space, we shall directly compare simulations with experimental observations.

\section{Experimental}

To explore the two-dimensional parameter space shown in Fig. 1, we have used two distinct experimental approaches. In so-called hybrid experiments, we increment the delay $\tau$ (or, equivalently, the cycle time $\tau_{\mathrm{c}}$ ) while keeping the number $2 n$ of refocusing pulses constant. This allows one to identify conditions where the echoes are not modulated. Once these favorable conditions have been found, echo decays are recorded by keeping $\tau$ constant and incrementing $n$, so that the apparent transverse relaxation times can be determined by fitting to a mono-exponential decay.

The measurements have been carried out with a sample of ${ }^{13} \mathrm{C}$ - and ${ }^{15} \mathrm{~N}$-enriched L-alanine (Cambridge Isotope Laboratories, $\mathrm{U}_{-}{ }^{13} \mathrm{C}_{3}, 98 \% ;{ }^{15} \mathrm{~N}, 98 \%$ ), $0.5 \mathrm{M}$ in $\mathrm{D}_{2} \mathrm{O}$ using a Bruker Avance spectrometer with a field $B_{0}=11.74 \mathrm{~T}$ (500 MHz for $\left.{ }^{1} \mathrm{H}\right)$. Fig. 2 shows experimental echo amplitudes $\mathrm{C}^{\alpha}{ }_{x}$ (blue) and simulated expectation values $\left\langle\mathrm{C}^{\alpha}{ }_{x}\right\rangle$ (red) in 


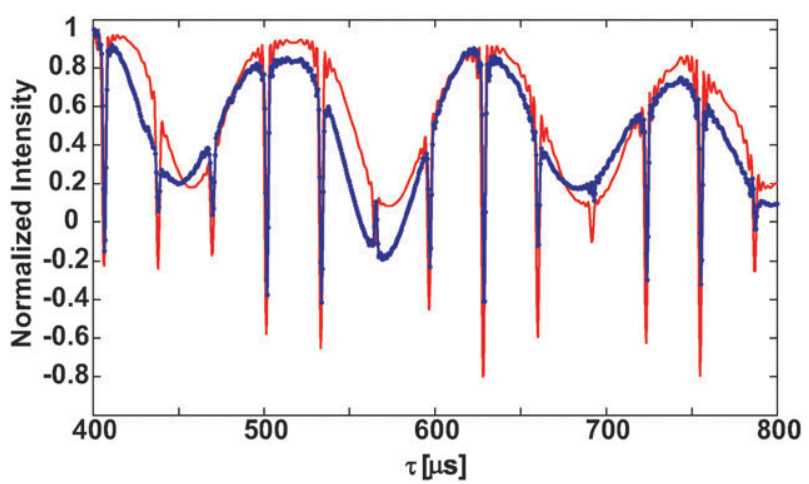

Fig. 2 Amplitudes $\left\langle\mathrm{C}^{\alpha}{ }_{x}\right\rangle$ of the 20th echo $(2 n=20)$ in ${ }^{13} \mathrm{C}$ - and ${ }^{15} \mathrm{~N}$-enriched alanine, as a function of the delay $\tau$. Experimental results are shown in blue, whereas simulated data are plotted in red. The ${ }^{13} \mathrm{C}$ $r f$ amplitude was $\omega_{1} /(2 \pi)=20 \mathrm{kHz}$, hence $\tau_{\pi}=25 \mu$ s. The $\tau$ delay has been varied between 400 and $800 \mu$ s in steps of $0.5 \mu \mathrm{s}$. The chemical shifts are $\delta\left(\mathrm{C}^{\alpha}\right)=50.37, \delta\left(\mathrm{C}^{\beta}\right)=16.02$ and $\delta\left(\mathrm{C}^{\prime}\right)=$ $175.76 \mathrm{ppm}$. At $B_{0}=11.74 \mathrm{~T}, \mathrm{C}^{\beta}$ and $\mathrm{C}^{\prime}$ appear at offsets of -4.32 and $15.77 \mathrm{kHz}$ with respect to $\mathrm{C}^{\alpha}$. The scalar coupling constants are $J\left(\mathrm{C}^{\alpha} \mathrm{C}^{\beta}\right)=35.0, J\left(\mathrm{C}^{\alpha} \mathrm{C}^{\prime}\right)=54.2, J\left(\mathrm{C}^{\beta} \mathrm{C}^{\prime}\right)=1.2, J\left(\mathrm{C}^{\alpha} \mathrm{H}^{\alpha}\right)=145.0$, $J\left(\mathrm{C}^{\beta} \mathrm{H}^{\alpha}\right)=4.4, J\left(\mathrm{C}^{\prime} \mathrm{H}^{\alpha}\right)=4.2, J\left(\mathrm{C}^{\beta} \mathrm{H}^{\beta}\right)=129.7, J\left(\mathrm{C}^{\alpha} \mathrm{H}^{\beta}\right)=4.6$ and $J\left(\mathrm{C}^{\alpha} \mathrm{N}\right)=5.7 \mathrm{~Hz}$.

alanine with $\Omega^{\alpha}=0$ and $\omega_{1} /(2 \pi)=20 \mathrm{kHz}$, as a function of the delay $\tau$, using a hybrid sequence with $2 n=20$. The superposition of two different recoupling conditions is clearly visible: the narrow dips, separated by small intervals $\Delta \tau^{\prime}$ are due to the $J\left(\mathrm{C}^{\alpha} \mathrm{C}^{\prime}\right)$ coupling to the spectrally remote $\mathrm{C}^{\prime}$ spin $\left(\Omega^{\prime} /(2 \pi)=15.77 \mathrm{kHz}\right.$, hence $\left.\gamma^{\prime}=0.79\right)$, while the broad dips, separated by large intervals $\Delta \tau^{\beta}$, are due to the $J\left(\mathrm{C}^{\alpha} \mathrm{C}^{\beta}\right)$ coupling to the $\mathrm{C}^{\beta}$ spin which has a chemical shift that is not far away from $\mathrm{C}^{\alpha}$, since $\Omega^{\beta} /(2 \pi)=-4.32 \mathrm{kHz}$, hence $\gamma^{\beta}=$ -0.22 . In order to determine the transverse relaxation rate $R_{2}{ }^{\text {app }}\left(\mathrm{C}^{\alpha}\right)$, it is necessary to find a flat region where the amplitude $A_{\text {mod }}$ of the modulations is small. In the range $400<\tau<800 \mu$ s, the best choice appears to be $\tau=515 \mu$ s.

To reduce the complexity of the problem, we can set the rf amplitude $\omega_{1}$ in such a manner that the refocusing pulses (which must act like $\pi$-pulses for the on-resonance $\mathrm{C}^{\alpha}$ spins) lead to a rotation through an angle $2 \pi$ for the off-resonance $\mathrm{C}^{\prime}$ magnetization. The effective nutation angle $\beta_{\text {eff }}$ is

$$
\beta_{\text {eff }}=\omega_{\text {eff }} \tau_{\pi}=\sqrt{\omega_{1}^{2}+\Omega^{2}\left(\mathbf{C}^{\prime}\right)} \tau_{\pi}
$$

which is equal to $2 \pi$ when

$$
\omega_{1}=\frac{\Omega\left(\mathrm{C}^{\prime}\right)}{\sqrt{3}} \approx 0.577 \Omega\left(\mathrm{C}^{\prime}\right)
$$

Fig. 3 shows that with this $r f$ amplitude the narrow recoupling conditions due to $J\left(\mathrm{C}^{\alpha} \mathrm{C}^{\prime}\right)$ have been suppressed as expected. However, a more complex pattern appears for the $J\left(\mathrm{C}^{\alpha} \mathrm{C}^{\beta}\right)$ interaction. This results from the fact that the $\mathrm{C}^{\beta}$ carbon is attached to three $\mathrm{H}^{\beta}$ protons, leading to a quadruplet. The four components, which have the usual binomial amplitudes $1: 3: 3: 1$, can be considered as four different effective offsets with respect to the $\mathrm{C}^{\alpha}$ carbon, leading to four different recoupling conditions.

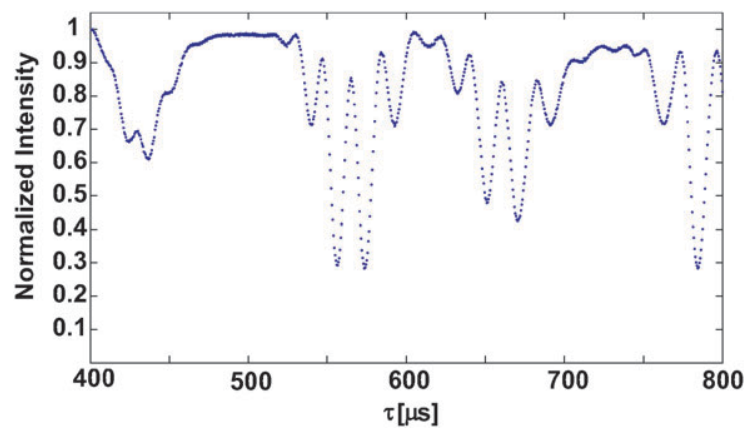

Fig. 3 Experimental echo amplitudes $\mathrm{C}^{\alpha}{ }_{x}$ in ${ }^{13} \mathrm{C}$ and ${ }^{15} \mathrm{~N}$ enriched alanine, observed with a hybrid sequence $\left[\tau-\pi_{x}-\tau\right]_{20}$ comprising $20 \pi$ pulses. The ${ }^{13} \mathrm{C} r f$ amplitude $\omega_{1} /(2 \pi)=9.1 \mathrm{kHz}$ corresponds to a $\pi$ pulse length $\tau_{\pi}=54.92 \mu$ s on-resonance $\left(\Omega^{\alpha}=0\right)$. This leads to a rotation about the tilted effective field $\left(\gamma^{\prime}=\Omega^{\prime} / \omega_{1}=1.73\right)$ through $2 \pi$ for the off-resonance $\mathrm{C}^{\prime}$ magnetization which has an offset $\Omega^{\prime} /(2 \pi)=$ $15.77 \mathrm{kHz}$. The $\tau$ delay has been varied between 400 and $800 \mu$ s in steps of $0.5 \mu \mathrm{s}$.

The regions where the echo amplitudes of $\mathrm{C}^{\alpha}{ }_{x}$ are not affected by modulations are quite narrow. Therefore it is not straightforward to measure precisely the apparent rate $R_{2}^{\text {app }}$ of $\mathrm{C}^{\alpha}$. A simplification could be obtained by decoupling the $\mathrm{H}^{\beta}$ protons during the multiple refocusing sequence. However, it has been shown previously ${ }^{9}$ that proton decoupling induces artefacts that prevent one from extracting reliable transverse relaxation rates.

Another approach to obtain unmodulated decays is to shift the carrier frequency $\omega_{r f}$ away from the $\mathrm{C}^{\alpha}$ resonance. Fig. 4 shows the experimental (blue) and simulated (red) effects of hybrid sequences on $\mathrm{C}^{\alpha}$ echoes when the carrier frequency is set exactly half-way between the $\mathrm{C}^{\prime}$ and $\mathrm{C}^{\beta}$ resonances. The experimental decays were still not totally free from modulations.

The amplitudes $A_{\text {mod }}$ of the $\mathrm{C}^{\alpha}$ echoes can be calculated as a function of the delay $\tau$ and of the carrier frequency, which was stepped over a range of $6 \mathrm{kHz}$ from the $\mathrm{C}^{\alpha}$ resonance towards the $\mathrm{C}^{\prime}$ resonance. The resulting map is shown in Fig. 5: dark blue areas correspond to regions where modulations are weak

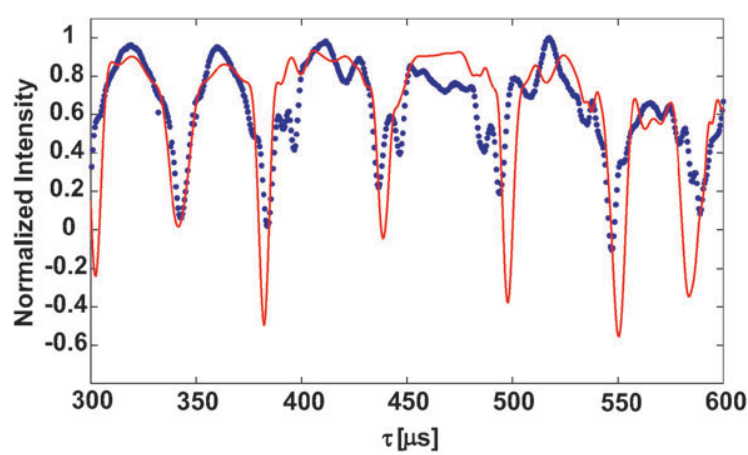

Fig. 4 Echo intensities of $\mathrm{C}^{\alpha}$ in alanine recorded with the hybrid sequence with $2 n=20$, the carrier frequency $\omega_{r f}$ being set half-way between the chemical shifts of the two coupling partners $C^{\prime}$ and $C^{\beta}$. The $r f$ amplitude was $\omega_{1} /(2 \pi)=19.4 \mathrm{kHz}$, hence $\tau_{\pi}=25.77 \mu \mathrm{s}$, $\gamma^{\prime}=0.81$ and $\gamma^{\beta}=-0.22$. The delays $300<\tau<600 \mu$ s were incremented in steps of $0.5 \mu$ s. Experimental results are shown in blue, whereas simulated data are plotted in red. 


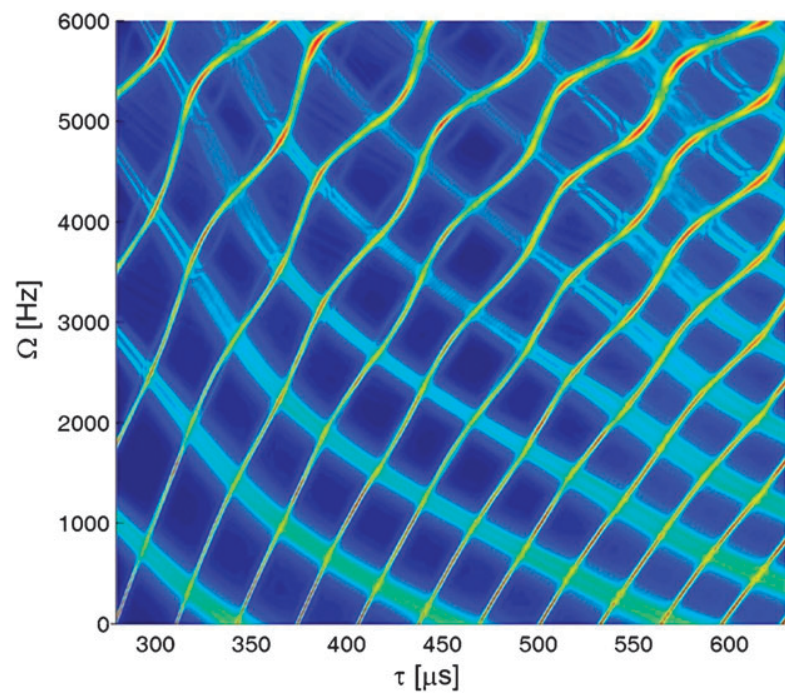

Fig. 5 Simulation of the amplitude $A_{\text {mod }}$ of the modulations of $\mathrm{C}^{\alpha}$ in alanine, with respect to the echo delay $\tau$ and the offset $\Omega$ separating the carrier frequency $\omega_{r f}$ from the chemical shift of the $\mathrm{C}^{\alpha}$ carbon. In the simulations, all three carbons $\left(\mathrm{C}^{\alpha}, \mathrm{C}^{\beta}\right.$ and $\left.\mathrm{C}^{\prime}\right)$ and four protons $\left(\mathrm{H}^{\alpha}\right.$ and $\left.3 \mathrm{H}^{\beta}\right)$ were taken into account. The $r f$ amplitude was $\omega_{1} /(2 \pi)=20 \mathrm{kHz}$. Dark blue areas correspond to regions without modulations, whereas yellow ridges indicate strong modulations.

( $A_{\text {mod }} \approx 0$ ), whereas yellow stripes indicate strong modulations $\left(A_{\bmod } \approx 1\right)$. The thin stripes with positive slopes correspond to $\mathrm{C}^{\alpha} \mathrm{C}^{\prime}$ recoupling, the broader stripes with negative slopes to the $\mathrm{C}^{\alpha} \mathrm{C}^{\beta}$ interactions. In the simulations, all three carbons $\left(\mathrm{C}^{\alpha}, \mathrm{C}^{\beta}\right.$ and $\mathrm{C}^{\prime}$ ) and four protons (one $\mathrm{H}^{\alpha}$ and three $\mathrm{H}^{\beta}$, but none of the $\mathrm{H}^{\mathrm{N}}$ protons which were replaced by deuterium) were taken into consideration. To verify the simulations, echoes were recorded at two points on the map featuring extreme values for the amplitude $A_{\text {mod }}$ of the modulations: at a crossing of two recoupling stripes (with an offset of $+1 \mathrm{kHz}$ from the $\mathrm{C}^{\alpha}$ chemical shift and a delay of $\tau=366.5 \mu \mathrm{s}$ ) and in the center of a "blue field" (with an offset of $+1.275 \mathrm{kHz}$ from the $\mathrm{C}^{\alpha}$ chemical shift and a delay of $\tau=391.25 \mu \mathrm{s})$. The resulting decays are shown in Fig. 6. In agreement with the simulations, the echo decay on the left,

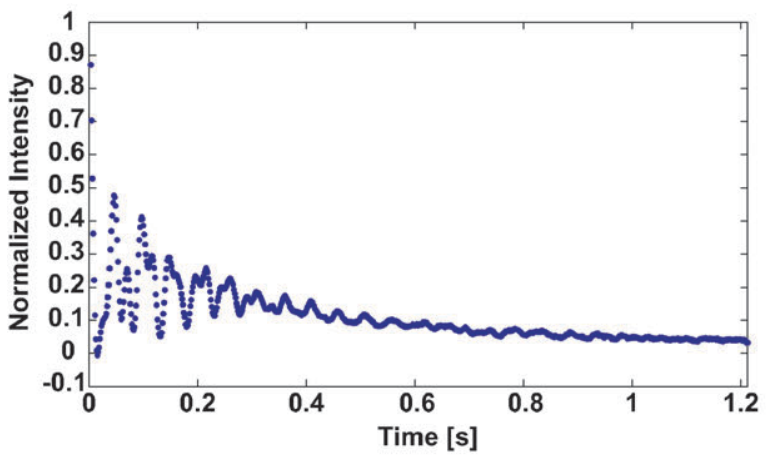

Table 1 Apparent transverse relaxation rates of $\mathrm{C}^{\alpha}$ in alanine, measured in regions where the amplitudes $A_{\text {mod }}$ of the modulations are small, according to the simulated map of Fig. 5

\begin{tabular}{llll}
\hline$\tau / \mu \mathrm{s}$ & $\begin{array}{l}\text { Offset/Hz of } \\
\omega_{r f} \text { w.r.t. } \mathrm{C}^{\alpha}\end{array}$ & $R_{2}{ }^{\text {app }} / \mathrm{s}^{-1}$ & $\begin{array}{l}\text { Simulated } \\
A_{\text {mod }}\end{array}$ \\
\hline 391.25 & 1275 & 0.48 & 0.034 \\
366.75 & 375 & 0.48 & 0.042 \\
416.50 & 1125 & 0.47 & 0.040 \\
467.50 & 475 & 0.51 & 0.046 \\
492.50 & 1225 & 0.50 & 0.035 \\
491.00 & 200 & 0.57 & 0.057 \\
517.00 & 975 & 0.52 & 0.052 \\
541.50 & 725 & 0.52 & 0.073 \\
566.50 & 500 & 0.51 & 0.091 \\
590.50 & 275 & 0.52 & 0.102 \\
467.50 & 1525 & 0.47 & 0.024 \\
\hline
\end{tabular}

recorded at a crossing of yellow stripes in Fig. 5, shows strong modulations leading to a fast decay of the echoes. On the other hand, the echo decay on the right is free of any modulations and can be perfectly fitted with a monoexponential function, leading to a transverse relaxation rate $R_{2}^{\text {app }}=0.48 \mathrm{~s}^{-1}$.

Transverse relaxation rates measured in other "blue fields" in the map of Fig. 5 are listed in Table 1, together with the corresponding amplitudes $A_{\text {mod }}$ of the modulations determined by simulations.

To verify that the resulting $R_{2}{ }^{\text {app }}$ rates are reliable, $1 \mathrm{M}$ natural abundance alanine and $0.01 \mathrm{M}$ uniformly enriched ${ }^{15} \mathrm{~N}-{ }^{13} \mathrm{C}$-alanine were mixed to guarantee the same $\mathrm{pH}$ and chemical exchange rates. The offset with respect to the $\mathrm{C}^{\alpha}$-resonance was $1.275 \mathrm{kHz}$ and $\tau$ was $391.25 \mu$ s. The 'true' relaxation rate in the natural abundance sample was $R_{2}^{\text {true }}=$ $0.389 \pm 0.004 \mathrm{~s}^{-1}$, and the 'apparent' relaxation rate in the enriched sample was $R_{2}^{\text {app }}=0.43 \pm 0.01 \mathrm{~s}^{-1}$. The difference $R_{2}{ }^{\text {app }}-R_{2}^{\text {true }}=0.04 \pm 0.01 \mathrm{~s}^{-1}$ is slightly too large to be explained by the dipolar relaxation to the neighbouring carbon-13 nuclei $\mathrm{C}^{\prime}$ and $\mathrm{C}^{\beta}$ that should contribute only about $0.01 \mathrm{~s}^{-1}$. It is possible that, in spite of our efforts, the modulations are still not completely suppressed, so that some unresolved residual couplings still contribute to broaden the lines and accelerate the echo decays.

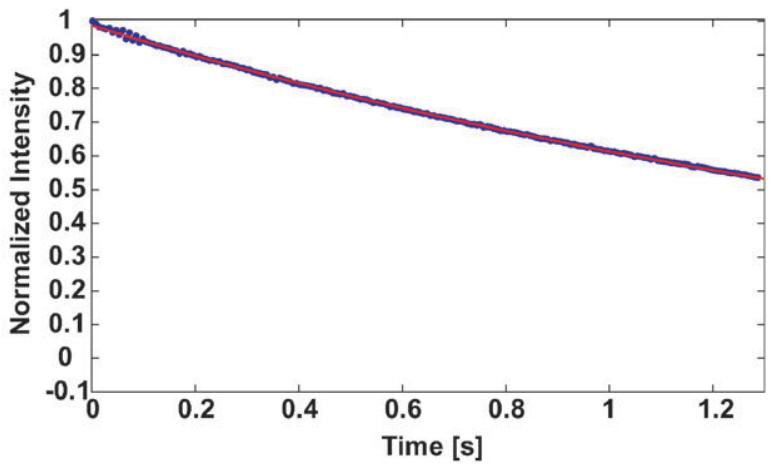

Fig. 6 Decays of $\mathrm{C}^{\alpha}$ echoes under a multiple refocusing sequence $(\pi / 2)_{y}-\left[\tau-\pi_{x}-\tau\right]_{2 n}$, incrementing $n$ from 1 to 800 in steps of 4 . The $r f$ amplitude was $\omega_{1} /(2 \pi)=20 \mathrm{kHz}$, corresponding to a $\pi$ pulse length $\tau_{\pi}=25 \mu$ s on resonance. The left decay was observed at a crossing of two yellow stripes in Fig. 5 with the carrier frequency at $+1 \mathrm{kHz}$ from the chemical shift of $\mathrm{C}^{\alpha}$ and $\tau=366.5 \mu$ s. The right decay was observed in a "blue field" of Fig. 5 with the carrier frequency at $+1.275 \mathrm{kHz}$ from the chemical shift of $\mathrm{C}^{\alpha}$ and $\tau=391.25 \mu$ s. Blue dots represent experimental data and red lines mono-exponential fits $I(t)=I_{0} \exp \left(-R_{2}{ }^{\text {app }} t\right)$ with $t=n\left(4 \tau+2 \tau_{\pi}\right)$. The transverse relaxation rate was determined to be $R_{2}{ }^{\text {app }}=$ $0.48 \mathrm{~s}^{-1}$. 


\section{Conclusions}

It has been shown that, despite the interference of homonuclear scalar couplings $J_{\mathrm{CC}}$, it is possible to measure reproducible apparent transverse relaxation rates $R_{2}{ }^{\text {app }}$ of carbon-13 nuclei. The echo decays in ${ }^{13} \mathrm{C}$ - and ${ }^{15} \mathrm{~N}$-enriched alanine are not modulated by $J_{\mathrm{CC}}$ couplings, provided one uses moderate $r f$ amplitudes that are comparable with the offsets of the coupling partners, so that their effective fields are tilted, and provided one selects suitable $\tau$ intervals, which can be readily identified in preliminary hybrid experiments where the number of echoes $n$ is kept constant and the delay $\tau$ is incremented. If on-resonance hybrid experiments do not reveal any useful quenching conditions, a variation of the carrier frequency may lead to conditions where the echo decays are smooth. After selecting a favourable delay $\tau$ and carrier frequency where echo modulations can be neglected, one can determine the apparent transverse relaxation rates $R_{2}{ }^{\text {app }}$ from simple mono-exponential fits.

\section{Acknowledgements}

We are indebted to Dr Jens Dittmer, Dr Karthik Gopalakrishnan, and Dr Bikash Bayshia for many fruitful discussions, and to
Martial Rey for valuable assistance. This work was supported by the Swiss National Science Foundation (FNSNF), the Ecole Polytechnique Fédérale de Lausanne (EPFL), the Swiss Commission for Technology and Innovation (CTI), and the CNRS (France).

\section{References}

1 J. Cavanagh, W. J. Fairbrother, A. G. Palmer, M. Rance and N. J. Skelton, Protein NMR Spectroscopy - Principles and Practice, Academic Press, San Diego, 2nd edn, 2007.

2 H. Y. Carr and E. M. Purcell, Phys. Rev., 1954, 94, 630.

3 S. Meiboom and D. Gill, Rev. Sci. Instrum., 1958, 29, 688.

4 J. Dittmer and G. Bodenhausen, J. Am. Chem. Soc., 2004, 126, 1314.

5 V. Y. Orekhov, D. M. Korzhnev and L. Kay, J. Am. Chem. Soc., 2004, 126, 1886

6 J. Dittmer and G. Bodenhausen, ChemPhysChem, 2004, 5, 1750.

7 J. Dittmer and G. Bodenhausen, ChemPhysChem, 2006, 7, 831.

8 K. Gopalakrishnan, N. Aeby and G. Bodenhausen, ChemPhysChem, 2007, 8, 1791.

9 N. Aeby and G. Bodenhausen, Chem. Phys. Lett., 2008, 463, 418.

10 B. Baishya, T. F. Segawa and G. Bodenhausen, J. Am. Chem. Soc., 2009, 131, 17538.

11 G. Bodenhausen, R. Freeman and G. A. Morris, J. Magn. Reson., 1976, 23, 171.

12 G. A. Morris and R. Freeman, J. Magn. Reson., 1978, 29, 433. 